\title{
Low-Noise Amplification, Detection and Spectroscopy of Ultra-Cold Systems in RF Cavities
}

\author{
Masroor H. S. Bukhari1 ${ }^{*}$, Zahoor H. Shah ${ }^{2}$ \\ ${ }^{1}$ Department of Physics, Jazan University, Gizan, Saudi Arabia \\ ${ }^{2}$ Department of Analytical Chemistry, Institute of Chemistry, University of Sindh, Jamshoro, Pakistan \\ Email: *mbukhari@jazanu.edu.sa,mbukhari@gmail.com
}

Received 15 March 2016; published 16 April 2016; published 20 April 2016

Copyright (C) 2016 by authors and Scientific Research Publishing Inc.

This work is licensed under the Creative Commons Attribution International License (CC BY).

http://creativecommons.org/licenses/by/4.0/

(c) (i) Open Access

\section{Abstract}

The design and development of a cryogenic Ultra-Low-Noise Signal Amplification (ULNA) and detection system for spectroscopy of ultra-cold systems are reported here for the operation in the 0.5 - $4 \mathrm{GHz}$ spectrum of frequencies (the " $L$ " and " $S$ " microwave bands). The design is suitable for weak RF signal detection and spectroscopy from ultra-cold systems confined in cryogenic RF cavities, as entailed in a number of physics, physical chemistry and analytical chemistry applications, such as NMR/NQR/EPR and microwave spectroscopy, Paul traps, Bose-Einstein Condensates (BEC's) and cavity Quantum Electrodynamics (CQED). Using a generic Low-Noise Amplifier (LNA) architecture for a GaAs enhancement mode High-Electron Mobility FET device, our design has especially been devised for scientific applications where ultra-low-noise amplification systems are sought to amplify and detect weak RF signals under various conditions and environments, including cryogenic temperatures, with the least possible noise susceptibility. The amplifier offers a 16 $\mathrm{dB}$ gain and a $0.8 \mathrm{~dB}$ noise figure at $2.5 \mathrm{GHz}$, while operating at room temperature, which can improve significantly at low temperatures. Both $d c$ and RF outputs are provided by the amplifier to integrate it in a closed-loop or continuous-wave spectroscopy system or connect it to a variety of instruments, a factor which is lacking in commercial LNA devices. Following the amplification stage, the RF signal detection is carried out with the help of a post-amplifier and detection system based upon a set of Zero-Bias Schottky Barrier Diodes (ZBD's) and a high-precision ultra-low noise jFET operational amplifier. The scheme offers unique benefits of sensitive detection and very-low noise amplification for measuring extremely weak on-resonance signals with substantial lownoise response and excellent stability while eliminating complicated and expensive heterodyne schemes. The LNA stage is fully capable to be a part of low-temperature experiments while being operated in cryogenic conditions down to about $500 \mathrm{mK}$.

${ }^{*}$ Corresponding author. 


\section{Keywords}

\section{Ultra Low-Noise Amplifier, VLNA, LNA, RF Spectroscopy, Microwave Spectroscopy, Weak Signal Detection}

\section{Introduction}

Weak signal detection is not only a substantial part of communications and network engineering, but also an important part of experimental physical sciences, where weak Radio Frequency (RF) measurements need to be performed on various physical systems, especially under stringent conditions of extreme temperatures. The first and foremost area is spectroscopy in physics and physical/analytical chemistry, where a weak signal from a sample or atomic/molecular system confined in an RF cavity is measured and its spectra are obtained. These include molecular microwave or rotational spectroscopy [1], Nuclear Magnetic Resonance (NMR) spectroscopy (and its variant Nuclear Quadrupole Resonance or NQR spectroscopy) [2], Electron Spin Resonance (ESR) or Electron Paramagnetic Resonance (EPR) Spectroscopy [3], etc. Other than the wide area of spectroscopy, some of the important contemporary fields of physical sciences, such as particle physics and condensed-matter physics, entail weak signal detection and spectral analysis from atoms or electrons in cavities. These experiments at times involve ultra-cold systems, such as atoms, molecules or Bose-Einstein Condensates (BEC's) [4] [5], kept at extremely low (sub-Kelvin) temperatures. Some of such applications include cavity Quantum Electrodynamics (CQED), resonance studies with resonators working at microwave frequencies [6], cavity-based experiments in particle physics, for instance, photon mass [7] and Dark Matter (DM) axion [8] [9] particles detection, which relies on detection of weak signals arising from resonance in cold RF resonant cavities, most often at cryogenic temperatures.

In astronomy and astrophysical applications [10] [11], ultra-low-noise cryogenic amplification of weak signals is sought in various RF bands from terrestrial or space-bound telescopy systems and astrophysical experiments. All these applications rely heavily on a low to ultra-low noise amplifier front-end which can offer a significant gain in signal power, while at the same time keeping the noise level to a minimum.

In connection with our studies on CQED and in the development of a spectroscopic technique (in low temperature regime), we needed a robust and extremely sensitive amplifier and RF signal detection system which could serve our purposes in a precision and low-cost manner. We found out that there was no such integrated system readily available in market which could offer the features we needed and third-party custom-built solutions were quite expensive. Thus we decided to design and develop an integrated ultra-low noise amplification and detection system which could aid in our research endeavors.

Low-Noise Amplifiers (LNA's) [12], especially narrow-band LNA's [13] [14], are well-known RF devices which are not only essential components of every RF communication or wireless system, but also find scope in spectroscopy techniques at microwave frequencies as well as in general-purpose RF weak signal detection, spectral analysis and general RF metrology. Out of these devices, there are certain designs which offer a very low noise amplification, satisfying the benchmark of noise figures around 0.5 to $1.0 \mathrm{~dB}$ at a few $\mathrm{GHz}$ with room temperature operation. These are known as Very/Ultra-Low-Noise Amplifier (VLNA/ULNA) devices which constitute an important part of RF engineering.

The design of a similar (but narrow-band) VLNA (which we call a cULNA, or cryogenic ULNA) amplification and detection system is documented in this report for weak signal detection applications in the $\mathrm{L}$ and $\mathrm{S}$ bands, specifically for scientific applications, offering the ability to amplify weak signals within any narrow ( $\sim 500 \mathrm{MHz}$ ) part of the spectrum from 1 to $4 \mathrm{GHz}$. In its essence, our amplifier is a general-purpose LNA design, which offers a stable amplification with quite low noise performance, in addition to signal detection and post amplification features, to detect and amplify weak RF signals at a few $\mathrm{GHz}$ frequencies. The amplifier design is aimed at optimal signal reproduction while preserving signal fidelity and with high noise immunity.

Our design is based upon a relatively newer generation GaAs Enhancement-mode pseudomorphic High-Electron Mobility Transistor (E-pHEMT/FET), the Avago ATF54143 [15], chosen for a number of important features such as excellent stability (as studies have demonstrated [16] [17]), associated gain of $16.6 \mathrm{~dB}$ and very low noise figure of $0.5 \mathrm{~dB} @ 2 \mathrm{GHz}$, and low-power consumption $(<2 \mathrm{~mW})$, while working at room temperature. 
Moreover, since it is made with GaAs material, it can operate down to a few Kelvins, as needed in low-temperature applications (such as CQED, circuit QED, quantum computation and cryogenic spectroscopy applications [18]). Unlike other specialized devices, this kind of HEMT is easily obtainable from any electronic supplier and is not expensive. We have customized the generic architecture of this design to act as a narrow-band device which can be customized for applications anywhere in the $0.5-4 \mathrm{GHz}$ band by changing the values of passive components [19] [20].

The device is coupled with a RF detection and post-detection amplification stage, which produces a signal which can be read by a Lock-in Amplifier (LIA) and/or a Data Acquisition (DAQ) scheme to detect the required signal and obtain time-domain data series as well as frequency-domain spectra (with the help of a Fast Fourier Transform, FFT, software). A significant benefit of this amplifier is production of an ordinary $d c$ voltage output for applications where resonance detection is sought, which cannot be obtained from off the shelf LNA devices available in the market. The commercial LNA devices are expensive as well, whereas this design can be made at half the cost, using components which are readily available from electronic distributors, such as Digikey (Thief River Falls, MN) in our case. Besides, the device could be used at ambient temperatures down to approximately $500-700 \mathrm{mK}$, with the use of special low thermal conductivity cables.

In short, we have endeavored to achieve the design and development of a very low noise RF amplifier and weak signal detector, which has potential to become an integral and central part of an experiment in RF spectroscopy or other physics/physical chemistry applications entailing low-noise amplification of a weak RF signal.

\section{The Amplifier, Detector and Post-Amplifier Design}

A general overview of our designed system is illustrated in the Figure 1, the block schematic, which contains three main sections. The first and crucial-most stage is the LNA section, which amplifies a weak RF signal presented at its input, under the control of a $d c$ Gate Bias Point (GBP) voltage from a feedback circuit, sensing the input signal $d c$ level and maintaining a suitable GBP. It produces two output signals, one is an RF high-frequency signal which can be read by a spectrum analyzer, for calibration and testing purposes, the other one is a dc output which can be passed to the next stage for resonance detection. The next stage is a detector, based upon two microwave frequency Zero-Bias Schottky Barrier Diodes (SBD/ZBD's), to sense the amplified ac signal and convert it into a corresponding $d c$ voltage. This stage also contains a passive low-pass filter network to eliminate spurious high-frequency signals picked up in the signal acquisition and amplification. The third and final stage is a high-precision post amplifier which amplifies the filtered and detected signal and presents it to the output for read-out. Adding a Lock-In amplifier (LIA) or a Data Acquisition System (DAQ/DAS) to the device turns it into a complete ultra-low-noise RF spectroscopy system.

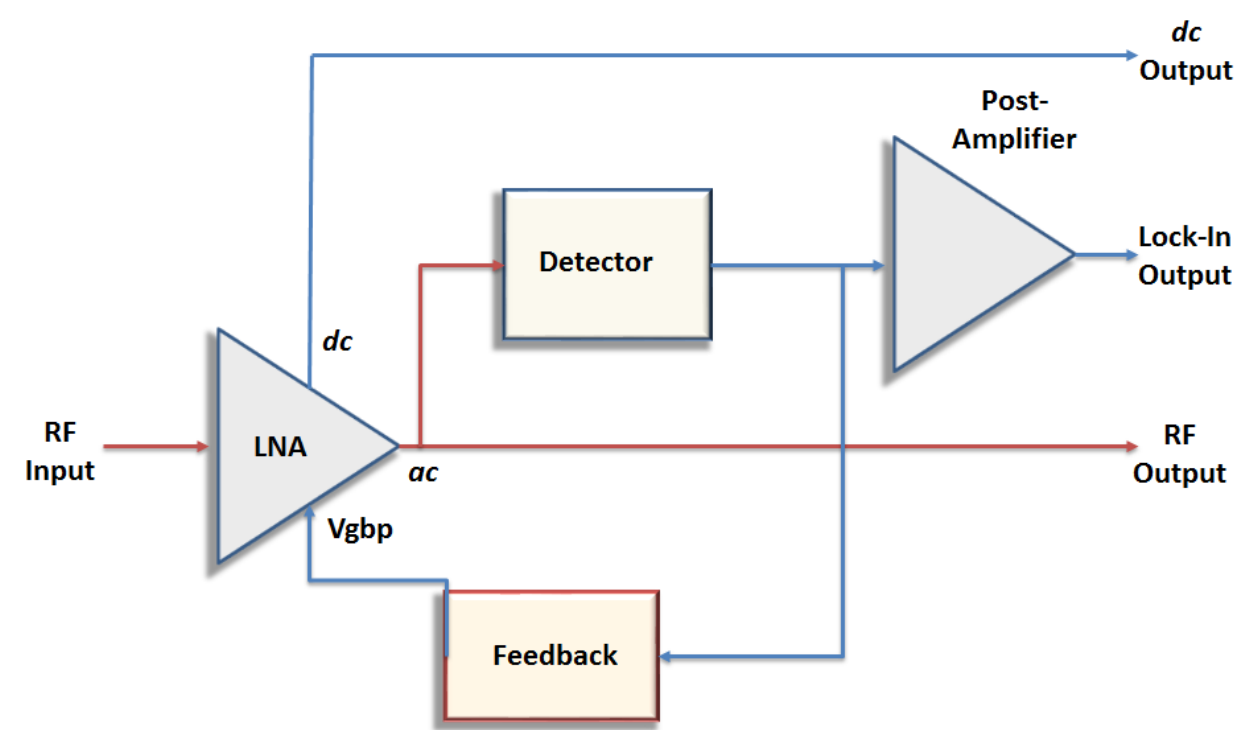

Figure1. A block schematic and overview of the detection system. Adding a LIA and DAQ can turn this into a complete RF spectroscopy system. 


\subsection{The Cryogenic Ultra-Low Noise Amplifier (cULNA)}

As illustrated in the Figure 2, the circuit schematic of the LNA device, the HEMT ATF54143 is at the heart of it, aided by a biasing scheme made around a general-purpose Bipolar Junction Transistor (BJT), Q2. The design is a generic LNA architecture for the Avago devices which is optimized here for the $\mathrm{S}$ and $\mathrm{L}$ bands, in view of the utility of these bands in various weak signal detection and RF spectroscopy methods. Majority of this circuit is a typical ATF HEMT LNA design, modified for our application, with the significant differences being the active biasing and $d c$ output signal extraction (in addition to an RF signal), and the special construction strategy and selection of passive components for the device (as outlined in the next section). The values of the LC networks used here have been devised for impedance matching and noise immunity for the 2 - $3 \mathrm{GHz}$ section, in order to obtain the best performance in this spectral region, in view of our application at hand. However, by varying the values of the LC components (C1, L2 and C4, L3), optimal noise reduction can be obtained at other frequencies as well, ranging from $200 \mathrm{MHz}$ to close to $4 \mathrm{GHz}$. The $d c$ signal obtained from the LNA section can directly be fed into a Lock-In amplifier or read-out into a DAQ system for on-line or offline signal and spectral analysis.

$\mathrm{L} 1$ is optional and recommended only for operation greater than $3 \mathrm{GHz}$ [19]. Conversely, for frequencies less than $1 \mathrm{GHz}$, as per the ATF54143 data sheet [15], each source pad of the HEMT needs to be strapped to the ground pad with a 0.040 " wide etch. Table 1 gives an approximate measure of our suggested values for the passive networks for various frequencies while using the same circuit. It has to be noted that these values are approximate and recommended in view of simulations and testing for best noise performance expected at the prescribed bands, but actual performance depends on a number of factors, such as the substrate used and the construction of the amplifier, etc.

Higher frequency operation is quite possible in similar manner but it would require a special substrate (unlike the usual PCB or copper clad board) in view of the special noise considerations at higher frequencies. In such

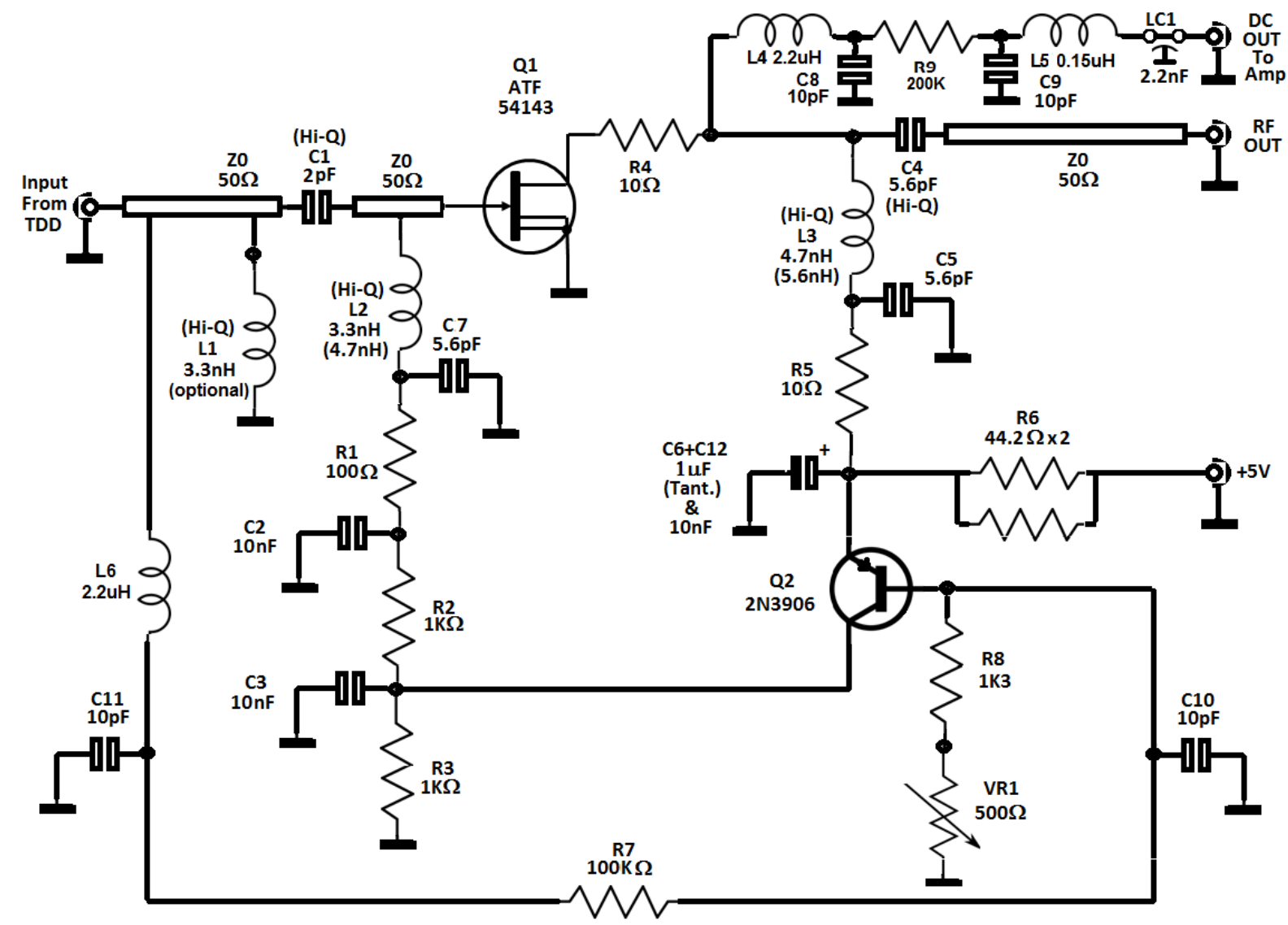

Figure 2. Circuit schematic of the ultra-low-noise-amplifier design as proposed here (the LC networks used in this design are optimized for the $2.0-2.6 \mathrm{GHz}$ band). 
Table 1. ULNA passive network values for various frequencies.

\begin{tabular}{ccccc}
\hline Frequency Range & $\mathbf{C 1}$ & $\mathbf{L 2}$ & $\mathbf{C 4}$ & $\mathbf{L}$ \\
\hline $\mathbf{0 . 4}$ to $1.2 \mathrm{GHz}$ & $8.2 \mathrm{pF}$ & $4.7 \mathrm{nH}$ & $8.2 \mathrm{pF}$ & $6.8 \mathrm{nH}$ \\
$\mathbf{1 . 3}$ to $1.9 \mathbf{~ G H z}$ & $8.2 \mathrm{pF}$ & $2.7 \mathrm{nH}$ & $8.2 \mathrm{pF}$ & $5.6 \mathrm{nH}$ \\
$\mathbf{2 . 0}$ to $2.6 \mathrm{GHz}$ & $2.4 \mathrm{pF}$ & $3.3 \mathrm{nH}$ & $5.6 \mathrm{pF}$ & $4.7 \mathrm{nH}$ \\
$\mathbf{2 . 7}$ to $3.0 \mathbf{~ G H z}$ & $2.2 \mathrm{pF}$ & $3.3 \mathrm{nH}$ & $5.6 \mathrm{pF}$ & $4.7 \mathrm{nH}$ \\
$\mathbf{3 . 1}$ to $3.9 \mathrm{GHz}$ & $1.5 \mathrm{pF}$ & $10 \mathrm{nH}$ & $3.6 \mathrm{pF}$ & $33 \mathrm{nH}$ \\
$\mathbf{4 . 0}$ to $4.9 \mathbf{~ G H z}$ & $1.0 \mathrm{pF}$ & $3.9 \mathrm{nH}$ & $3.0 \mathrm{pF}$ & $3.9 \mathrm{nH}$ \\
$\mathbf{5 . 1}$ to $5.5 \mathrm{GHz}$ & $0.8 \mathrm{pF}$ & $3.9 \mathrm{nH}$ & $2.2 \mathrm{pF}$ & $3.9 \mathrm{nH}$ \\
\hline
\end{tabular}

cases a PTFE (Teflon) board with copper clad may be a suitable choice. The values of resistors in the design are chosen for the operation of the HEMT at its optimal voltage bias point. We have chosen the active bias scheme, for which a general purpose PNP bipolar junction transistor, Q2, the 2N3906 (or MMBT3906 the SMD version) has been utilized. VGBP is the HEMT Gate Bias Point (GBP) voltage which can to be obtained from a constant voltage source (which is $+5 \mathrm{~V}$ in this case), or for a more precision operation, obtained from a feedback loop, as we have suggested in the block schematic. In such a situation, the loop circuit would maintain this bias point by monitoring the $d c$ input voltage presented at the RF input. The feedback loop can be implemented with the help of a precision inverting comparator with a precision voltage reference driving the HEMT through Q2.

Working at its optimal bias point, the HEMT, Q1, takes an RF input and amplifies it to a higher level and presents an RF output via the $d c$ block capacitor C4. This output is used with an SMA connector using a $50 \Omega$ coaxial cable. However, before this $d c$ block, the signal has a $d c$ voltage which is pulled out via a low-pass filter network (comprising L4, C8, C9, R9, C5 and a feed-through LC filter LC1), to present to a Lock-in amplifier or a data acquisition system. For cryogenic operation, such as on the fingers of a dilution refrigerator, special low thermal conductivity cables would replace the coaxials, and feed-through low-pass filters would need to be added to all incoming and outgoing leads (except the power) from the LNA cold box.

\subsection{The Detector and Post-Amplifier}

The next section is the post-LNA amplifier and detection unit, as illustrated in the Figure 3, integrating the detector diodes with it. The detector part comprises a full-wave diode detection scheme using a pair of Avago HSMS-2852 matched zero-bias microwave Schottky Barrier Diodes (SBD's) [21], which are perhaps the most sensitive and best RF detectors in our knowledge for this application (or any low-signal RF application). The pair of diodes serves as a robust detector and an AM demodulator, producing a voltage signal corresponding to the incoming RF ac signal (It should be noted here that for lower frequencies, a pair of lower cost and more widely available ST Microelectronics 1N5711 Schottky diodes could also be used). If the input signal is of sufficient high-intensity, a $20 \mathrm{~dB}$ attenuator is mandatory before the ZBD stage. Alternatively, the sensitive and vulnerable HSMS285X can be replaced by the high-signal HSMS282X series SBD's, without making any changes in the circuit, especially for operation above $1.5 \mathrm{GHz}$ frequencies (since the HSMS285X can only work up to that limit). A pertinent replacement for HSMS2852 is the BAT15 ZBD's from Infineon, which could be employed in this application, however we did not test those. It has to be noted that the HSMS285X series has a maximum inverse voltage rating of $2.0 \mathrm{~V}$. To protect ZBD's from breakdown, it has to be assured that there should not be development of a voltage of more than $1.5 \mathrm{~V}$ around these devices.

The amplification stage was originally designed and developed around the high-precision AD745 ultra-low noise high-speed BiFET operational amplifier from Analog Devices (Analog Devices, Norwood, MA). AD745 offers ultra-low noise features (a very low voltage noise of $2.9 \mathrm{nV} / \mathrm{VHz}$ at $10 \mathrm{kHz}$ ) and THD of $0.0002 \%$ @ 1 $\mathrm{kHz}$ and excellent ac performance with Gain Bandwidth (GBW) product of $20 \mathrm{MHz}$ and a slew rate of $12.5 \mathrm{~V} / \mu \mathrm{s}$. But, later on, in view of not getting the satisfactory results, which could have been because of the problems with our design and not necessarily owing to the device, a Texas Instruments TLE2071 low-noise jFET op-amp device [22] was employed in the next prototype, though with degradation in noise performance and bandwidth (it has $12 \mathrm{nV} / \mathrm{rtHz}$ maximum noise floor at $1 \mathrm{KHz}$ and a $10 \mathrm{MHZ}$ GBW). In order to increase the gain and sensitivity of the amplifier, there is a cascode FET stage before the U1 comprises a pair of low-noise 2N4416A FETs 


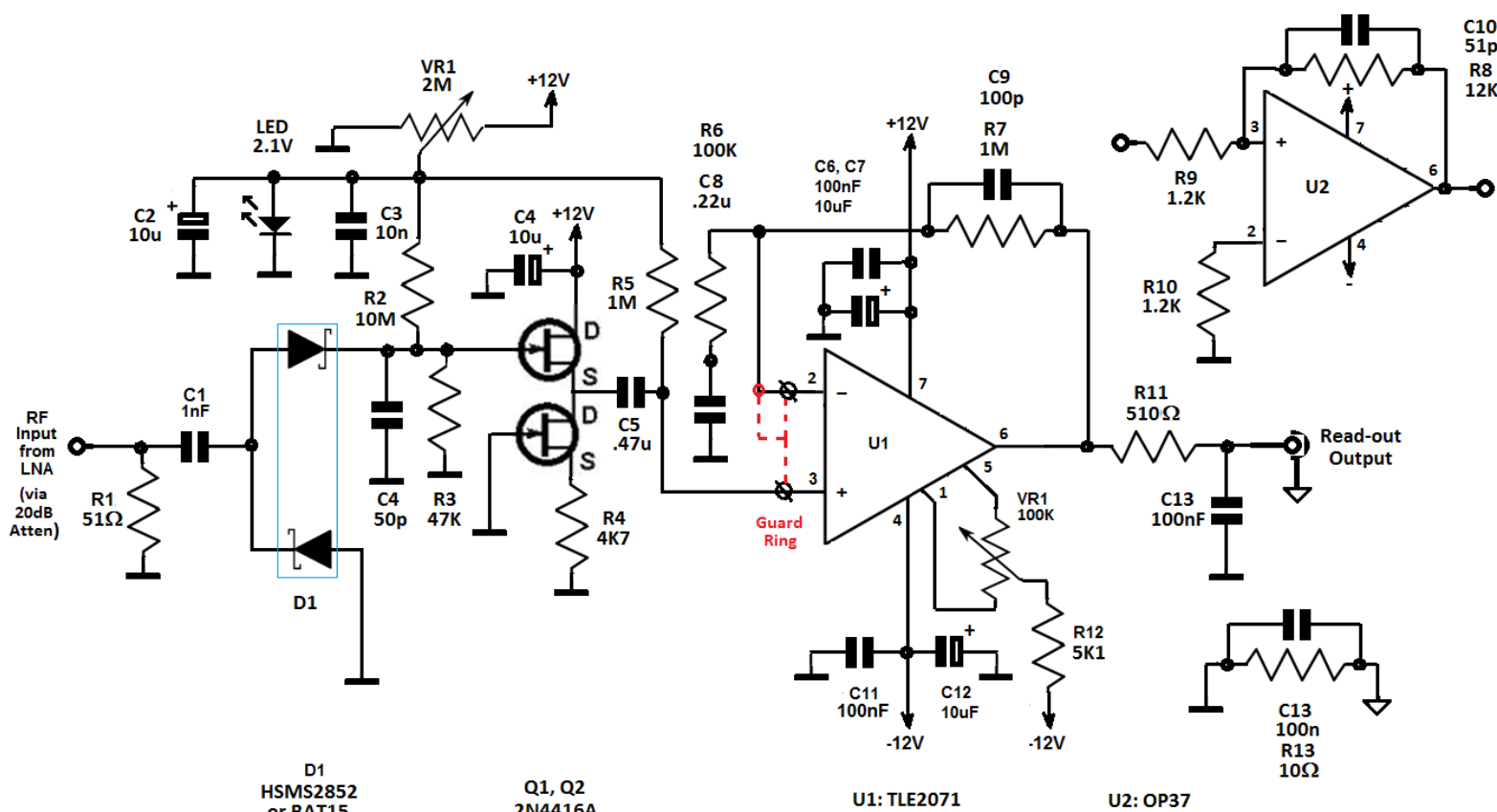

Figure 3. A block schematic of the zero-bias Schottky Barrier Diode (SBD) detection system following the LNA stage, based upon HSMS-2850 ZBD's and a precision low-noise RF post-amplifier built around a precision TLE2071 low noise high-speed jFET operational amplifier. An optional 250 KHz Low-Pass Filter (LPF), based on OP37/OP27 shown in the inset, can be added to output for optimal signal processing.

(we used the Fairchild MMBF4416, the SMD version, with lowest noise specs). This stage is optional and can be removed, connecting ZBD directly to the non-inverting input of U1. There is a stable bias network formed with the help of VR1, C2, LED and C3 to provide bias voltage for the amplifier. The value of VR1 should be set around $100 \mathrm{~K}-150 \mathrm{~K}$. The feedback circuit around the U1 is carefully chosen to compensate for the input signal conditions around the Schottky detectors and to reduce the measurement error. Two additional Schottky detectors (back to back) can be employed here in the feedback circuit of the U1 for further amplification of the RF signal at the input. The VR2 trimmer with U1 is used for setting the offset point for the amplifier. An optional Low-Pass Filter (LPF) stage, centered around $260 \mathrm{KHz}$, is provided with the help of U2 (Analog OP37), if required, before presenting the signal to a Lock-In Amplifier (LIA) or a read-out device. The C13, R13 RC network is for protection of the circuit from the output instrument.

A regulated power supply unit was made to obtain the $\pm 12 \mathrm{~V}$ and $+5 \mathrm{~V}$ dc regulated power for the detector and ULNA. Battery operation was decided to obtain the best noise performance for the evaluation and application of this design. A farm of batteries was used to obtain $\pm 18 \mathrm{~V}$ source power which was converted into the required power, using LM78L12, LM79L12 and KA7805 regulator IC's for $+12 \mathrm{~V},-12 \mathrm{~V}$ and $+5 \mathrm{~V}$ output, respectively.

\section{Construction}

A substantial part of the LNA/ULNA performance lies in the implementation of its design. Construction of the amplifier, starting from the selection of appropriate substrate (i.e. a copper clad or PCB) and associated passive components all the way to their appropriate placement and careful assembling of the device, require special design considerations and assiduous handling and assembling practices.

Building the ULNA is the most crucial stage of construction, requiring special RF considerations and it required building of a number of prototypes and damaging a lot of components, till the successful operation could become possible. In order to save the cost, for our initial prototypes we used a 2-sided 1oz. $0.79 \mathrm{~mm}(0.031$ ”) FR4 copper clad board (Digi-key part number 473-1010-ND), which was cut into a size of $42 \mathrm{~mm} \times 40 \mathrm{~mm}$, and covered with a layer of solder on both side, keeping one side for mounting components and other for theground plane. Both sides were clamped to each other by copper shims soldered on both sides along the perimeter. 
We built the amplifier using a "Dead-bug" scheme, without a PCB design, by mounting the components directly on the board on one another or via small copper shims or solder wick pieces and using Teflon stand-off' $\mathrm{s}$ or Teflon tape for insulation where required. Appropriate vias were drilled in the board for optimal RF operation. Figure 4(a) illustrates a view of salient components and vias, as well as connectors. All the components used are SMD (except C6, R6 and R7) and were soldered before soldering the HEMT. A grounded general-pur- pose 15W soldering iron with 1/32" tip seemed to be the best choice for soldering all SMD components, with the help of a 28SWG (0.015”) Sn- $\mathrm{Pb}(60$ - 40) solder wire. Stringent static precautions are extremely crucial, as the HEMT is particularly vulnerable to damage by electrostatic discharge. All the inductors used in the design were Hi Q type, we found better results with wire wound ones among those (we used the Wurth WE-KI series and the Abracon AISC-0805 series in our evaluations). The capacitors used in the design are general-purpose chip capacitors (Murata GRM series) except the small-value $\mathrm{pF}$ capacitors which are the microwave, low-loss, RF high frequency type with high Q value (we built our prototypes with the AVX MLO and UQ series capacitors). Resistors used were $1 \%$ tolerance $100 \mathrm{ppm} /{ }^{\circ} \mathrm{C}$ thick film type (Yageo RC series or the Bourns CR0402 series). All components were carefully placed and soldered on one side of the board and the other was left untouched.

The completed board was then housed in a $5.5 \mathrm{~mm}$ thickness heavy gauge extruded aluminum enclosure (with sandblast anodized finish and built-in grooves to hold the board, and offering superior electromagnetic shielding performance), inner dimensions (LWH) $45 \mathrm{~mm} \times 39.7 \mathrm{~mm} \times 12.9 \mathrm{~mm}$ (Diligent Electronics Co. Ltd., China, www.aliexpress.com), with its inner dimensions coinciding tightly with the board's width and length. A tightlyfitted resolute enclosure assures best operation and helps eliminateself oscillations in the ULNA. PCB-soldered SMA female jacks are used to connect $50 \Omega$ signal coaxial cables to and from the LNA via male SMA connectors. For the DC output an ordinary coaxial cable is used with SMA male and BNC male connectors at its two ends. The complete unit is illustrated in Figure 4(b) and Figure 4(c).

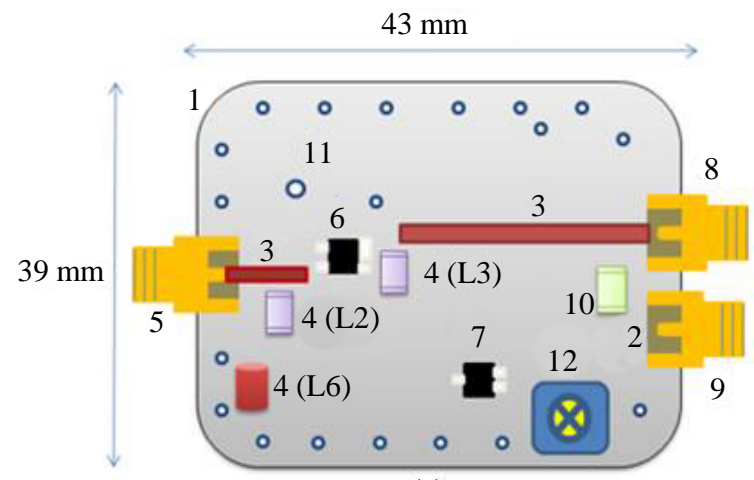

(a)

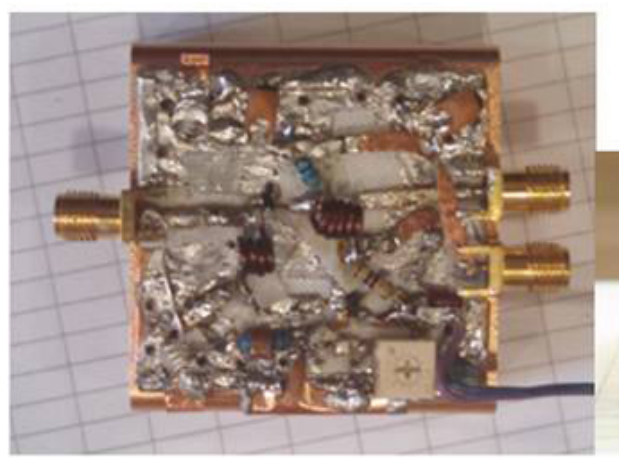

(b)

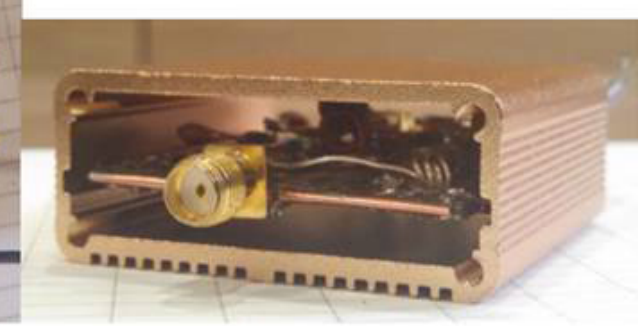

(c)

Figure 4. a) The suggested layout for the ULNA board. 1. FR4/PTFE Board, 2. Teflon Strip, 3. $50 \Omega$ Micro-strip, 4 . Inductors (this prototype used hand-wound inductors which were later replaced in following prototypes with HiQ SMD inductors), 5. SMA Connector, 6. HEMT transistor, 7. BJT transistor, 8. RF OUT, 9. DC OUT, 10. F/T LC Filter, 11. Via Holes, 12. Potentiometer, b) A photograph of our prototype; c) Cross sectional view of the unit while mounted in the case. 
As per the norms of RF design, all connections have to be made short (especially the ground connections, which must be less than $3 \mathrm{~mm}$ ) and multiple ground loops have to be eradicated. There should be no sharp angles in the path of the signal, especially at the input and output of the HEMT. Any wires in our out of the circuit must not be loose and have to be anchored to avoid triboelectric and other spurious effects.

The detector section was made on a 2-sided copper clad FR4 prototype PCB board on a grounded copper sheet plane across the perimeter of the board (where all the ground connections were made) and enclosed in an enclosure similar to the ULNA, but with twice the size. It does not require any special considerations or as stringent considerations and care like the building of the ULNA, but nevertheless careful layout, proper grounding and soldering need to be performed, avoiding ground loops and other issues. Static precautions are mandatory here as well, in view of the FET Op-Amp and other sensitive components, such as the SBD's.

\section{Testing and Results}

The electrical performance of the LNA and post-amplifier was tested in our experiment platform for detecting resonance in a home-made pick-up antenna tuned with a $2.5 \mathrm{GHZ}$ signal. The amplifier was operated with batteries and the amplifier and sample chamber were mounted in a grounded Faraday cage (with an extra $70 \mathrm{~dB}$ RFI and EMC shielding fabric around it from Aaronia GmBH) on an anti-vibration table. HEMT was operated while maintaining its bias point at approximately $3.0 \mathrm{~V}$.

The test gear used to test and calibrate the device consisted an Aaronia Spectran 6060V5 $6 \mathrm{GHz}$ Spectrum Analyzer and Agilent/KeysightN9310 3 GHz Function Generator for RF studies, whereasa Measurement Computing (MCC) 16-Bit 1.33MSPS MCC-1604AO Data Acquisition system and Agilent DSOX 200 MHz Digital Storage Oscilloscope were employed for $d c$ and mixed-signal studies. RF noise measurements are performed with the help of a generic noise meter (Agilent/Keysight N8973A 10MHz-3 GHz Noise Meter). If needed, further phase and gain testing can be performed with an Analog Devices AD8032 RF/IF Gain and Phase Detector evaluation module to measure gain and phase separately (which we are currently building in-house).

Figure 5 illustrates the noise figure performance of the LNA, where one can see a $0.6-0.8 \mathrm{~dB}$ noise figure in the frequencies of interest $(2.0-3.0 \mathrm{GHz})$ with the values of components chosen in our version. Figure 6 plots a calibration curve for the amplifier, depicting amplifier output voltage as a function of detected signal strength. Figure 7 illustrates a signal detected from a $2.5 \mathrm{GHZ}$ low-power source at a distance of $0.25 \mathrm{~m}$ from the detecting antenna.

Noise Figure

(at Room Temp)

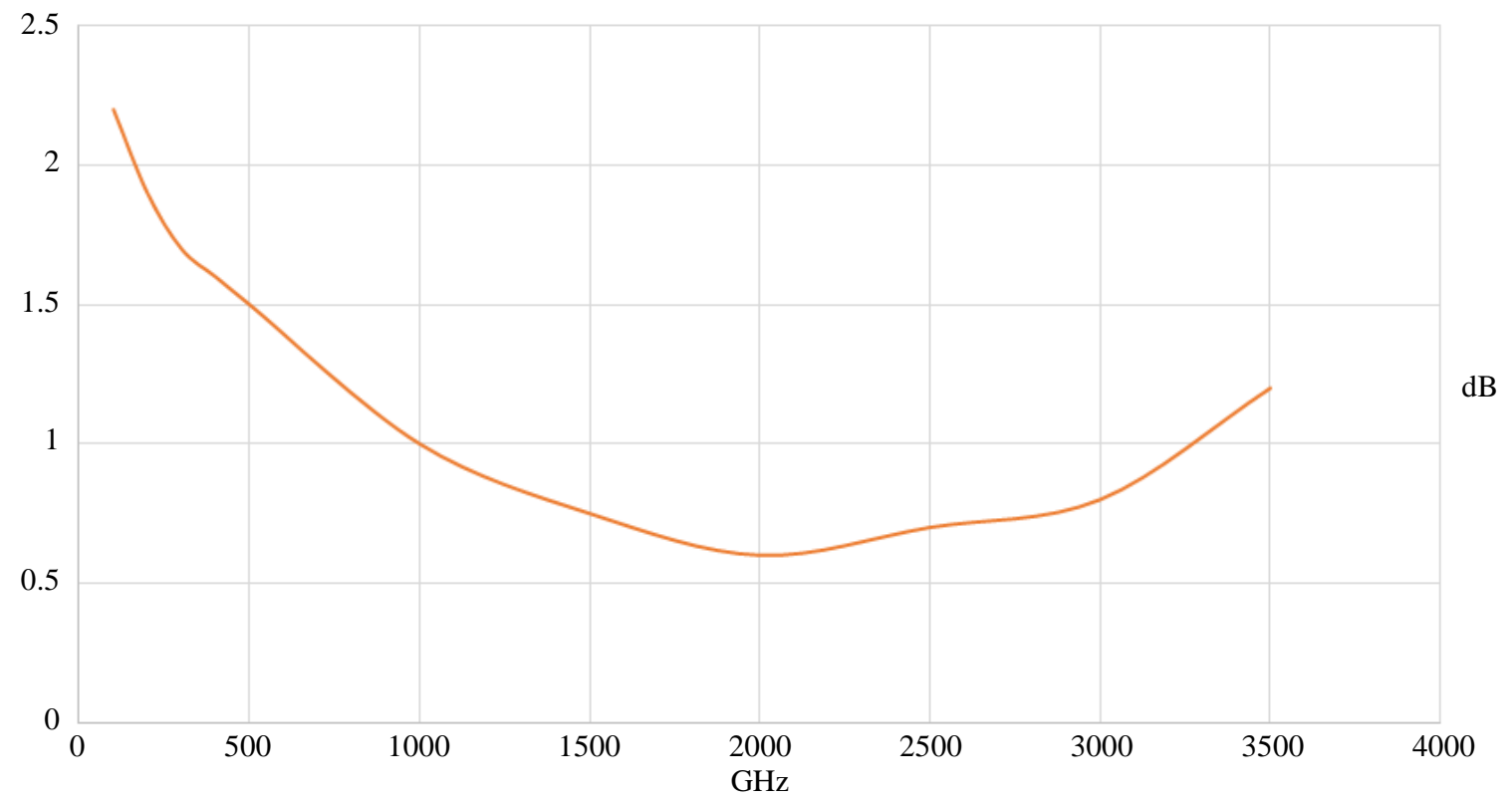

Figure 5. Noise figure (at room temperature). 


\section{Voltage vs Field Strength}

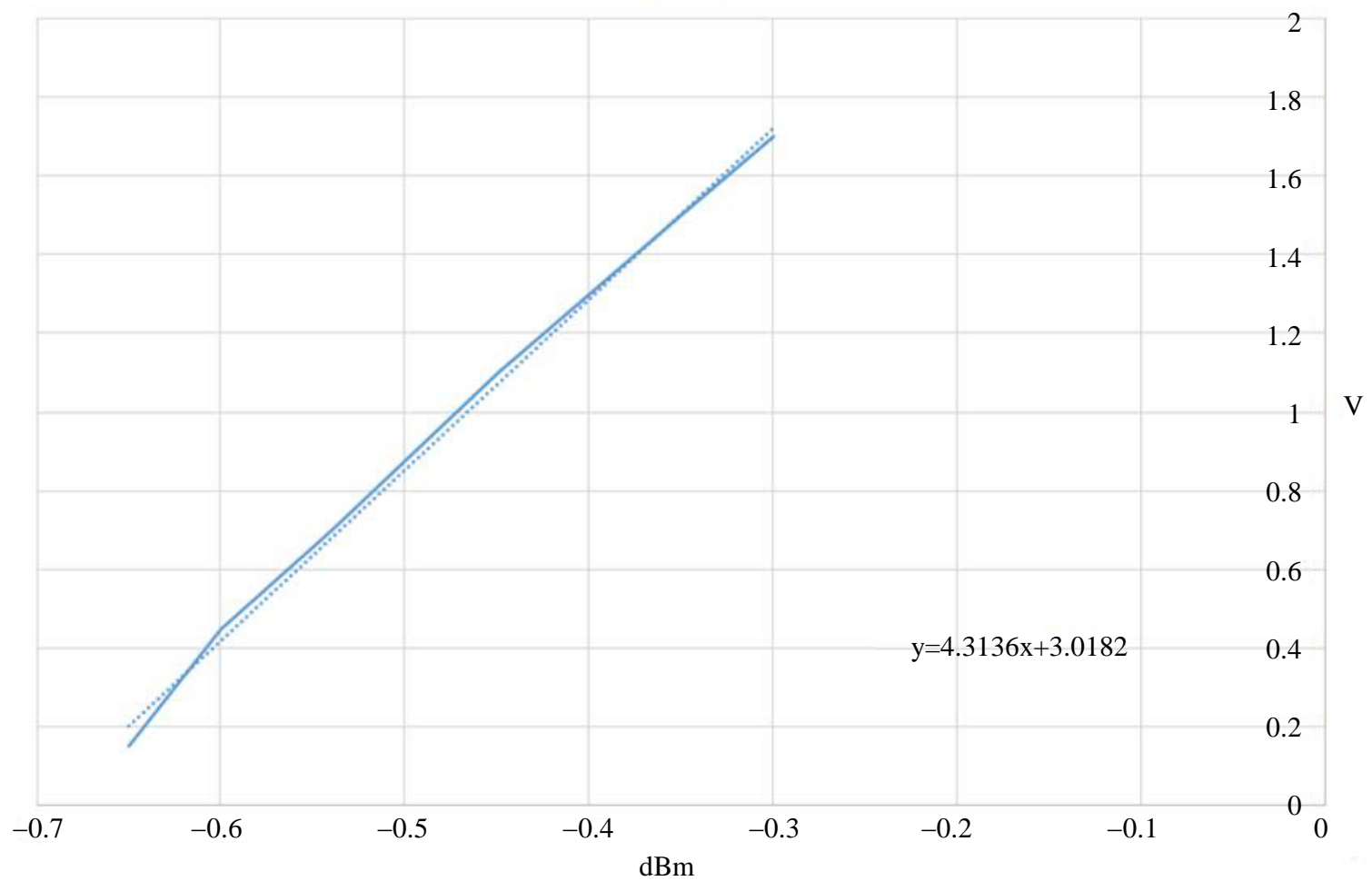

Figure 6. Amplifier output voltage vsdetected signal power (calibration curve).

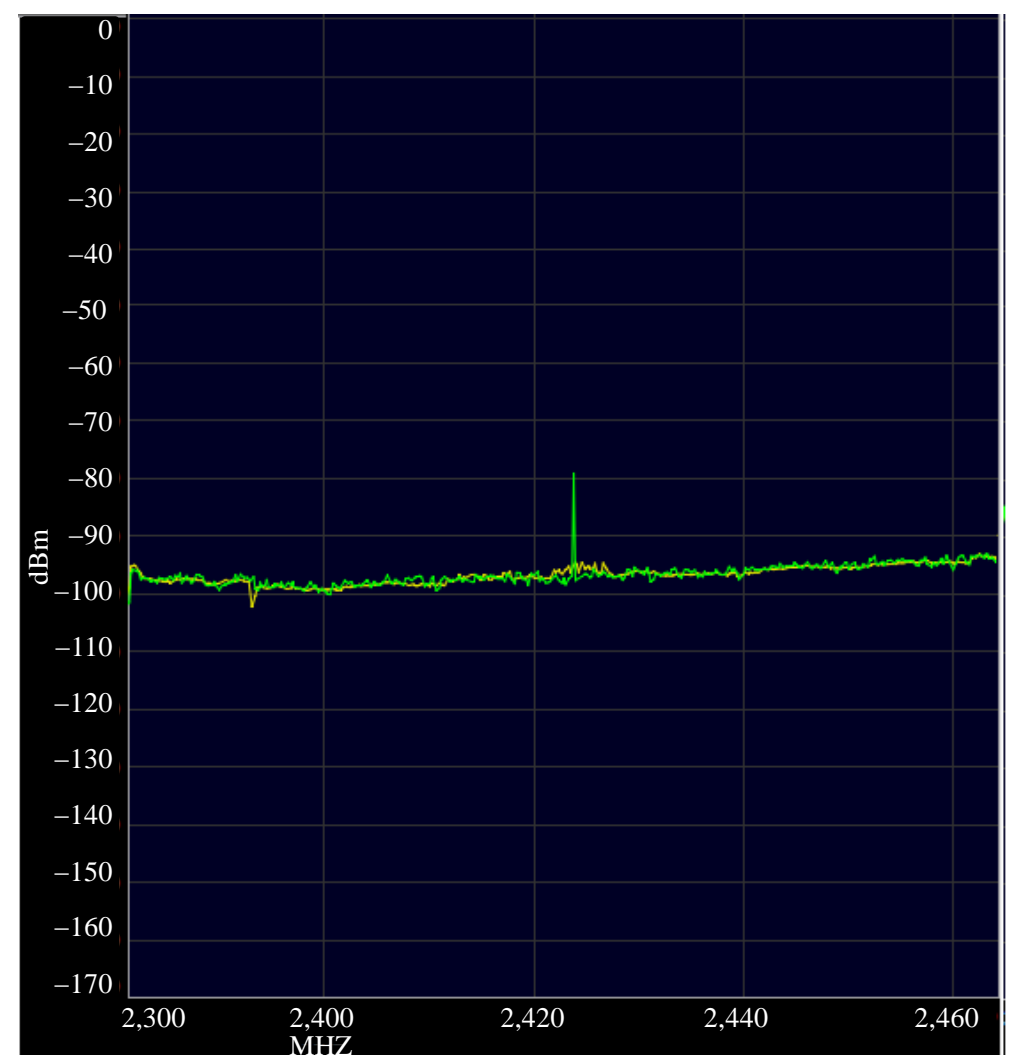

Figure 7. A detected signal from a test $2.5 \mathrm{GHZ}$ source in air at a $0.25 \mathrm{~m}$ distance. 
The amplifier demonstrated a sufficiently low noise figure of around $0.6-0.8 \mathrm{~dB}$ and a noise floor of approximately $-100 \mathrm{dBm}$ at $2.0 \mathrm{GHz}$ (while operated at room temperature), which is a satisfactory result. This translates to a noise temperature of approximately $55-57 \mathrm{~K}$ (at the $\mathbf{T}_{\text {phys }}$ of room temperature) but we estimate that in the low temperature regime $(100 \mathrm{mK}-2 \mathrm{~K})$, where its operation is desirable in cryogenic studies, it could drop down significantly, touching a lower limit of approximately 5 - $10 \mathrm{~K}$. Further drop is possible with more careful construction considerations and using two such amplifiers in a cascade configuration, which should bring the noise temperature to the design's limit of around $2 \mathrm{~K}$. This should find great applications for the amplifier in particle physics, radio astronomy and spectrometry.

In order to test the viability of the design in a scientific application of cavity QED spectroscopy, we tested the amplifier in the application of magnetic resonance spectroscopy to detect a resonant signal from water and ethanol as a result of EPR or NMR resonance, while sweeping a stimulating RF field of $50-100 \mathrm{MHz}$ from our signal generator. We made a custom grounded cavity and kept a vial of pure Ethanol $\left(\mathrm{CH}_{3} \mathrm{CH}_{2} \mathrm{OH}\right)$ mixed with water $\left(\mathrm{H}_{2} \mathrm{O}\right)$, encapsulating the sample in a home-made custom pick-up coil (made with 23SWG copper wire) while in the presence of a uniform magnetic field. The magnetic field was obtained by means of a Halbach geometry magnets array (made with four ND52 14,800 G $19 \times 19 \times 19 \mathrm{~mm}^{3}$ rare earth NdFeB permanent magnets [23]) to give a uniform central field of $1.48 \mathrm{~T} \pm 0.2 \mathrm{~T}$. The cavity was irradiated with a train of $100 \mathrm{MHz}$ pulses from signal generator. After a number of adjustments in the detection setup a signal was finally obtained from the sample as a result of resonance, as shown in Figure 8.

Further testing and development of an application using this platform is under way in the detection of ultraweak signals at low temperatures from a high-Q value RF cavity in an experimental particle physics application [24].

\section{Conclusions}

This is a preliminary report presenting the design of an LNA/ULNA and detection scheme for detection of weak signals arising from resonance corresponding to various physical phenomena and for general RF spectroscopy in the $S$ and $L$ bands. Both the LNA and post-amplifier proved to be extremely sensitive, not just in their performance, but also to their surroundings, requiring stringent grounding and EMI shielding for their optimal operation and utility.

High associated gain, very low noise figure, ability to operate at low temperatures and very low power consumption, make it a useful amplification and detection platform for a spectrum of RF applications in scientific instrumentation. Building of the amplifier entails careful construction considerations in order to obtain the desired operation and precision and best possible utility in the applications where it is sought. Although we made

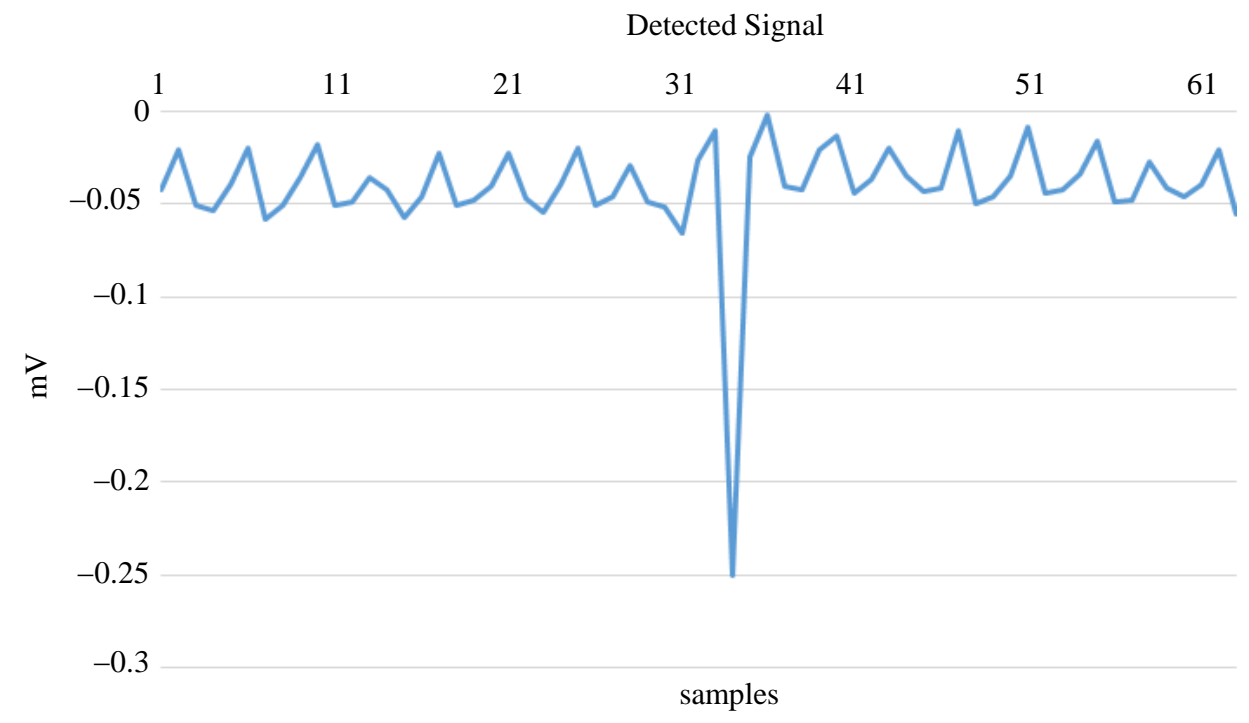

Figure 8. Detected resonant signal from a suspension of $\mathrm{CH}_{3} \mathrm{OH}$ and $\mathrm{H}_{2} \mathrm{O}$ with a $100 \mathrm{MHz}$ source signal in a $1.48 \mathrm{~T} \pm 0.1 \mathrm{~T}$ magnetic field, as recorded with the DAQ in time-domain. 
substantial care in designing and assembling the amplifier (making a number of successive prototypes), like in every endeavor, there is plenty of room for improvement. Using a pair of these HEMT's in a cascode configuration can make higher improvements in noise and construction with a special substrate that could extend the frequency range of the LNA amplifier.

Further improvement in gain and noise performance is quite possible by using better technologies than GaAs materials, such as In Asor In PHEMT devices [25]. Such LNA devices have been built by a number of astrophysical collaborations, such as NRAO [26] and the Planck probe, for astrophysical applications in the form of stand-alone, dual or cascode configurations [26] [27]. Since these are not available commercially, it is not possible for us to acquire and evaluate such a device, however, if our resources permit, we would like to test this design with such a device.

\section{Acknowledgements}

Funding support from the Deanship of Scientific Research (JU DSR Grant Number 3159), Jazan University, Saudi Arabia, the King Abdul Aziz City of Science and Technology (KACST) Small Grant (Grant number 234 35), and a faculty grant from the Department of Physics, Faculty of Science, Jazan University, are acknowledged with gratitude.

\section{References}

[1] Barrow, R.F., Long, D.A. and Sheridan, J., Eds. (1973) Molecular Spectroscopy, Vol. I. RSC Publishing, London.

[2] Keeler, J. (2010) Understanding NMR Spectroscopy. 2nd Edition, Wiley, Hoboken.

[3] Weil, J.A. and Bolton, J.R. (2006) Electron Paramagnetic Resonance: Elementary Theory and Practical Applications. 2nd Edition, Wiley, Hoboken. http://dx.doi.org/10.1002/0470084987

[4] Cornell, E.A. and Wieman, C.E. (1998) The Bose-Einstein Condensate. Scientific American, 278, 40-45.

[5] Jochim, S., et al. (2003) Bose-Einstein Condensation of Molecules. Science, 302, 2101-2103.

[6] Kumar, N. and Gupta, S.C. (2014) A Planar Microstrip Metamaterial Resonator Using Split Ring Dual at Ku-Band. International Journal of Physical Sciences, 9, 26-33. http://dx.doi.org/10.5897/IJPS2013.4088

[7] Bukhari, M.H.S. (2014) A Phenomenological Model for Photon Mass Generation in Vacuo. International Journal of Physical Sciences, 9, 48-53. http://dx.doi.org/10.5897/IJPS2013.4025

[8] Asztalos, S.J., Carosi, G., Hagmann, C., Kinion, D., van Bibber, K., Hotz, M., et al. (2010) SQUID-Based Microwave Cavity Search for Dark Matter Axions. Physical Review Letters, 104, 041301-041305.

[9] Dutra, S.M. (2005) Cavity Quantum Electrodynamics: The Strange Theory of Light in a Box. Wiley, Hoboken.

[10] Shurakov, A., Seliverstov, S. Kaurova, N. Finkel, M. Voronov, B. and Goltsman, G. (2012) Input Bandwidth of Hot Electron Bolometer with Spiral Antenna. IEEE Transactions on Terahertz Science and Technology, 2, 400-405. http://dx.doi.org/10.1109/TTHZ.2012.2194852

[11] Hesler, J.L. and Crowe, T.W. (2007) Responsivity and Noise Measurements of Zero-Bias Schottky Diode Detectors. In: Karpov, A., Ed., Proceedings of the 18th International Symposium on Space Terahertz Technology, ISSTT Publishing, Pasadena.

[12] Gonzalez, G. (1984) Microwave Transistor Amplifiers Analysis and Design. Prentice-Hall, Englewood Cliffs.

[13] Lee, T.H. (1989) The Design of Narrow-band CMOS RF Low-Noise Amplifiers, In: Huijsing, J., de Plassche, R.V. and Sanssen, W., Eds., Analog Circuit Design, Springer, New York, 303-316.

[14] Radmanesh, M.M. (2007) RF and Microwave Design Essentials, Engineering Design and Analysis From DC to Microwaves. Authorhouse, New York.

[15] (2012) ATF-54143 Low Noise Enhancement Mode Pseudomorphic HEMT in a Surface Mount Plastic Package Datasheet. Document Number AV02-0488EN, Avago Technologies, San Jose.

[16] Payne, K. (2009) Practical RF Amplifier Design Using the Available Gain Procedure and the Advanced Design System EM/Circuit Co-Simulation Capability. Agilent Technologies Document Number 5990-3356EN, Agilent Technologies, San Jose.

[17] Zhao, X.R., Fan, H.H., Ye, F.Y., Qian, X.F., Chen, D. and He, S. (2015) Design of a Two Stage Low Noise System in the Frequency Band 1.8-2.2 GHz for Wireless System. International Journal of Future Generation Communication and Networking, 8, 111-122.

[18] Silverman, M.P. (2000) Probing the Atom: Interactions of Coupled States, Fast Beams, and Loose Electrons. Princeton University Press, Princeton. 
[19] (2002) Low-Noise Amplifiers using ATF55143 Low-Noise PHEMT. Application Note 1285, Agilent Technologies, Agilent, San Jose.

[20] (2007) A 3.3 GHz to 3.8 GHz 802.16a WiMAX LNA using Avago Technologies ATF-54143. Application Note 5331, Avago, San Jose.

[21] (2009) HSMS-285x. Surface Mount RF Zero-Bias Schottky Barrier Diodes Data Sheet. AV02-1377EN, Agilent Technologies, Avago, San Jose.

[22] (2009) TLE207X Ex-Calibur Low-Noise High-Speed JFET-Input Operational Amplifiers Data Sheet. Document Number SLOS181C, Revised Edition, Texas Instruments, Dallas.

[23] K \& J Magnetics (2015) BCCC-N52 14,800 G NdFeB Magnet Data Sheet. K \& J Magnetics, Pipersville.

[24] Bukhari, M.H.S. and Shah, Z.H. A Detection Scheme for Resonant Cavity-based Dark Matter Searches.

[25] Schleeh, J. (2012) Ultra-Low Noise InP HEMTs for Cryogenic Amplification: Engineering Licenciate Dissertation. Chalmers University of Technology, Goteborg.

[26] Samoska, L.A., et al. (2011) Cryogenic MMIC Low Noise Amplifiers for W Band and Beyond. In: NRAO ISTT Proceedings, Charlottesville, 193-196. http://search.space-thz.org/catalog/2011193196

[27] Bryerton, E.W., et al. (2009) A W-Band Low Noise Amplifier with 22K Noise Temperature, IEEE Microwave Symposium Digest. IEEE MTT-S International, Charlottesville, 7-12 June 2009, 681-684. 